\title{
Decision aids against overuse of orthodontic services?
}

\section{Alexander Spassov, Bernard Braun, Sofija Carceva-Salja, Hartmut Bettin \& Dragan Pavlovic}

To cite this article: Alexander Spassov, Bernard Braun, Sofija Carceva-Salja, Hartmut Bettin \& Dragan Pavlovic (2017): Decision aids against overuse of orthodontic services?, Journal of Orthodontics

To link to this article: http://dx.doi.org/10.1080/14653125.2017.1282207

\section{曲 Published online: 30 Jan 2017.}

Submit your article to this journal $₫$

a

View related articles $\sqsubset$

View Crossmark data $₫$ 
LETTER TO THE EDITOR

\title{
Decision aids against overuse of orthodontic services?
}

\author{
Alexander Spassov ${ }^{\mathrm{a}}$, Bernard Braun ${ }^{\mathrm{b}}$, Sofija Carceva-Saljac, Hartmut Bettin ${ }^{\mathrm{a}}$ and Dragan Pavlovic ${ }^{\mathrm{d}}$

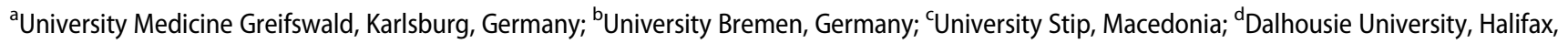 \\ Canada
}

\section{Letter to editor on:}

Development and evaluation of a patient decision aid for young people and parents considering fixed orthodontic appliances by Marshmann et al. (2016). J Orthod. 43:276-287.

The development of the first fixed appliance decision aid (FADA) may be an enormous advancement in improving the quality of orthodontic care (Marshmann et al. 2016). The importance of the work arises especially from the fact that decision aids may facilitate the shared decision-making process by means of providing up-todate scientific evidence on benefits and harms if presented in an appropriate format and at a right time point during the patient-physician communication. That is to say, through better information and better understanding, FADA may strengthen patient autonomy which is the fundament of bioethics and professional ethics.

As we read through the study however, we have certain doubts that authors conclusion that the FADA provides patient-relevant information, a hallmark of decision aids, probably cannot be drawn from this study. According to the criteria of the International Patients Decision Aids Standards (IPDAS), relevant treatment options presented in the decision aids have to include also the 'comprehensive, critically appraised, and up-to-date syntheses of the scientific evidence' (IPDAS 2012). However, according to the section Methods of the study, the authors did not conduct any systematic review and critical appraisal of the available evidence to inform the content of FADA. For example the FADA's content on root resorption, white spot lesions and patient experience were supported by only few selected single studies without clear selection criteria (Marshmann et al. 2016). Yet if the FADA fails to include some up-to-date and critically appraised evidence it may misinform patients (ethical dimension), cause unnecessary treatments (quality of care dimension) and thus resulting in more harm than good (Montori et al. 2013). We are aware of the difficulties associated with providing up-to-date scientific evidence for available treatment options, often limited funding, time consuming appraisal of literature, or low quality of available systematic reviews, just to mention a few. However, those limitations should be explicitly mentioned in the study so readers may be aware of them when drawing conclusion from the results. Also, strategies have been suggested to overcome the limitations of traditional decision aids and they could be useful in developing the FADA (Agoritsas et al. 2015).

To conclude, the authors made a first and probably most important step toward strengthening patient autonomy, given that the content and the presentation of the FADA is truly evidence-based. In orthodontic care, where probably misinformation and subsequent excessive overtreatment are common (Ackermann 2010; Spassov et al. 2015), decision aids may be one valuable strategy to overcome those problems that may causes unnecessary harm and costs to patients and society.

\section{Literature}

Ackerman MB. 2010. Selling orthodontic need: innocent business decision or guilty pleasure? J Med Ethics 36:275278.

Agoritsas $T$, Heen AF, Brandt $L$, Alonso-Coello $P$, Kristiansen $A$, Akl EA, Neumann I, Tikkinen KA, Weijden Tv, Elwyn G, et al. 2015. Decision aids that really promote shared decision making: the pace quickens. BMJ. 350:g7624.

International Patient Decision Aid Standards (IPDAS) Chapter K. 2012. Available from: http://www.ipdas.ohri.ca/IPDASChapter-K.pdf (accessed December 2016).

Marshman Z, Eddaiki A, Bekker HL, Benson PE. 2016. Development and evaluation of a patient decision aid for young people and parents considering fixed orthodontic appliances. J Orthod. 43:276-287.

Montori VM, LeBlanc A, Buchholz A, Stilwell DL, Tsapas A. 2013. Basing information on comprehensive, critically appraised,

CONTACT Alexander Spassov alexspas@uni-greifswald.de E Institute of Pathophysiology, University Medicine Greifswald, Greifswalder Str. 11c, 17495 Karlsburg, Germany 
and up-to-date syntheses of the scientific evidence: a quality dimension of the International Patient Decision Aid Standards. BMC Med Inform Decis Mak. 13 (Suppl 2):S5.

Spassov A, Bettin H, Pavlovic D. 2015. From misinformation to overtreatment. Am J Orthod Dentofacial Orthop. 147:295.

\section{Dear Dr Spassov and colleagues}

Thank you very much for your praise of our recent paper (Marshman et al. 2016) and for the opportunity to describe in more detail how we incorporated evidence into the Fixed Appliance Decision Aid (FADA).

We acknowledge that in the method section of our paper we simply stated that the FADA was informed by clinical evidence and cited the references we used. The development of the FADA was undertaken as a PhD and more details about how the FADA was developed can be found in the thesis, including how the evidence was searched for and appraised (Eddaiki 2015).

The published version of the FADA was developed and tested in 2013, using best practice at that time (Elwyn et al. 2006). In addition to the clinical effectiveness data, the evidence-base to inform the content of this decision aid was generated during its development process to ensure it was able to meet the needs of patients and parents. Qualitative interviews with children and parents, to identify the aspects of decision-making about fixed orthodontic treatment of importance to them, are a key aspect of patient decision aid development (Coulter et al. 2013). Systematic searches of the literature were conducted of clinical effectiveness evidence to identify the benefits and risks of treatment, including patient reported outcomes measures such as satisfaction with the outcome of orthodontic treatment, the prevalence of demineralised lesions and root resorption. The hierarchy of evidence was used to select papers for use in the FADA and the quality of these studies was assessed using the Quality Assessment Tool for Studies with Diverse Designs (QATSDD) (Sirriyeh et al. 2012). In the thesis there is a discussion of the quality of the included evidence and the completed QATSDD tool for each paper is included in the appendices. The thesis is available online with open access and we hope you find therein the detail to answers your remaining questions.

As you note, identifying accurate evidence for inclusion in patient decision aids is a key component of their rigour. Many patient decision aids draw on the evidence identified and synthesised in Cochrane, Health Technology Assessment and other systematic reviews of treatment effectiveness. We are not aware of any systematic reviews that would alter the current evidencebased within the FADA, and this is certainly an important direction of future research for our field and practice. The clinical effectiveness evidence base will continually need to be reviewed as the FADA is updated for future use.

Like you, we were delighted to find that (a) providing patients with accessible and accurate information structured in a way to support active reasoning about treatment options enabled patients to engage with their care more effectively, and (b) this type of patient resource can be integrated within current orthodontic practice. The next step is to look at its impact on clinical effectiveness and patient benefit.

\section{Disclosure statement}

No potential conflict of interest was reported by the authors.

\section{References}

Coulter, A., Stilwell, D., Kryworuchko, J., Mullen, P.D., Ng, C.J., Van Der Weijden, T., 2013. A systematic development process for patient decision aids. BMC Med Inform Decis Mak. 13 Suppl 2, S2.

Eddaiki, A. 2015. From compliance to concordance in orthodontic treatment: development of a patient decision aid for children considering fixed orthodontic appliance treatment [PhD thesis]. Available from: http://ethos.bl.uk/OrderDetails. do?uin=uk.bl.ethos.675045

Elwyn G, O'Connor A, Stacey D, Volk R, Edwards A, Coulter A, Thomson R, Barratt A, Barry M, Bernstein S, et al. 2006. International Patient Decision Aids Standards (IPDAS) Collaboration: developing a quality criteria framework for patient decision aids: online international Delphi consensus process. BMJ. 333 (7565): 417. doi:10.1136/bmj.38926. 629329.AE

Marshman Z, Eddaiki A, Bekker HL, Benson PE. 2016. Development and evaluation of a patient decision aid for young people and parents considering fixed orthodontic appliances. J Orthod. 43:276-287.

Sirriyeh R, Lawton R, Gardner P, Armitage G. 2012. Reviewing studies with diverse designs: the development and evaluation of a new tool. J Eval Clin Pract. 18: 746-752 